\title{
SAIL-AERODYNAMIC WIND POWER STATION WITH AUTOMATICALLY CHANGING BLADE-SWEPT AREA
}

\author{
BUKTUKOV N.S ${ }^{\mathbf{1}}$, BUKTUKOV B.ZH ${ }^{\mathbf{2}}$, MOLDABAYEVA G.ZH ${ }^{\mathbf{3}}$ \\ ${ }^{1}$ RK Academy of Sciences; Mining Institute after D.A. Kunayev, Almaty, Kazakhstan \\ ${ }^{2,3}$ Mining Institute after D.A. Kunayev, Almaty, Kazakhstan
}

\begin{abstract}
The wind power station with high utilization ratio over time, rated power utilization ratio and efficiency ratio has been developed. It works efficiently at wind speeds ranging from 3 to $50 \mathrm{~m} / \mathrm{sec}$. At the same time, the rated power is achieved at speeds ranging from $5-8 \mathrm{~m} / \mathrm{sec}$ to $50 \mathrm{~m} / \mathrm{sec}$.

KEYWORDS: Wind power station with automatically changing blade-swept area, Utilization ratio over time \& Rated power utilization ratio
\end{abstract}

Received: Apr 17, 2020; Accepted: May 08, 2020; Published: Jun 04, 2020; Paper Id.: IJMPERDJUN202081

\section{INTRODUCTION}

The WSEC-2017 Congress of Scientists and Engineers "Energy of the Future: Innovative Scenarios and Methods for Their Implementation" held in the framework of the EXPO-2017 International Exhibition on June 19-20 in Astana showed ever-increasing importance of Wind Power Stations (WPS) and solar panels. This scientific field has been recognized as a promising one. In addition, regulatory acts are being adopted that promote the development of renewable energy sources [1,6-16]. At the same time, the rated power utilization ratio and the utilization ratio of wind power stations over time do not ensure the reduction of cost of generated electricity to the cost of energy from mains. In addition, there is also an environmental problem. All these issues reduce demand.

It is possible to solve the problem by effective operation of wind power stations in a wide range of wind speeds and the exclusion of negative environmental impacts, namely infrasound. And this can be achieved by creating and using low-speed wind power stations with automatically changing blade-swept area that decreases at increasing wind speed and increases at decreasing wind speed.

In the world practice, there were attempts to expand the range of operating wind speeds [2] by adjusting revolutions or power, and by using a vertical shaft that eliminates the impact of wind direction changes on the utilization ratio of wind power stations over time. The controlled movement takes place due to the centrifugal force or auxiliary drive.

However, the movement of the load over the bus causing a change in the blade-swept area is not always possible. For example, foreign objects brought in by wind or snow onto the bus as well as water and freezing of water in the cold season will hinder the movement of the load on the bus, which will lead to breakdown of the installation at high wind speeds. If the bus is covered with a corrugated hose, frozen water in corrugations will not allow the hose to compress. Consequently, the load will not move along the bus, which will also lead to breakdown of the installation at strong winds. In addition, there is knocking between the load and the bus and due to which 
wind pressure on the blades will deflect the load toward the bus.

To increase power, it is necessary to expand the blade-swept area, including blades area (semicircular rims). And this leads to the decrease in the rotation speed of the wind power unit, as a result of which centrifugal force significantly reduces and the load does not move along the bus.Therefore, there will be no control of excess power.

In addition, two blades (semicircular rims) uncontrollably close under the influence of wind when the wind direction coincides with the line passing through the axis of rotation between the vertical edges of two opposite semicircular rims. This leads to jerky rotation of the wind power plant, which causes premature destruction of bearings of the main shaft and uneven load on the generator.

Automatic limitation of the rotational speed [3] allows the centrifugal force to deform internal blades in order to reduce the cross-sectional area of the air flow path through the rotor.

However, in the position when the blades are perpendicular to the direction of wind, the air does not get inside the blades, and the installation is supposed to pass this section of the rotation path inertially. Therefore, the power take-off to the generator will reduce the rotation speed, so the rotation will be jerky. As a blade-swept area increases, including blades area, to increase power, the rotation speed is reduced; the inertia energy is not enough to overcome the rotation resistance created by the generator. The uniformity of rotation can be achieved by increasing the number of blades, but in this case, this is not possible.

There is an option [4] where the turbine consists of blades mounted around a central axis so that they can rotate together around it. At that, each turbine blade is mounted on the axis towards its outer periphery around the axis, mainly parallel to the central axis. The offset device shifts the inner periphery of the turbine blades towards the central axis. When the turbine blades are placed in an air stream, they rotate together around the central axis. And as the rotation speed increases, they rotate around the support contrary tothe shift, resulting in a decrease in the rotation speed.

Here are the same drawbacks as in [2]. Moreover, there appears a knock between the load and the shaft, since the wind pressure on the blades will deflect the load towards the shaft. In this case, the translational movement of the load along the shaft can be eliminated by removing the shaft between the supports of the blades. However, in this case, the uncontrolled movement of the load will prevent the blades from opening and closing simultaneously. This reduces the efficiency of rotation speed control and can lead to breakdown of the installation in strong winds. In addition, to augment the power, it is necessary to increase the parameters of the blades as well as to lengthen the connections, which leads to a rise in the diameter of the circle circumscribed by the supports. In this case, the rotation speed (number of revolutions) is reduced, and the centrifugal force cannot deflect the blades (racks) and lift the load, which makes it impossible to control the rotation speed. In addition, the shift of the center of gravity of the turbine blades from the axis of rotation towards the end of the connection also prevents the development of centrifugal force if the diameter increases.

However, this problem can be solved in the following way. Since sail stations are more efficient at low wind speeds, and aerodynamic ones at medium speeds, it is necessary to integrate sail and aerodynamic blades of WPS. In addition, it is necessary to make provision for uninterrupted operation of the wind power station at high wind speeds.

Such an attempt was made in [5] (Figure 1) with some design improvements. 


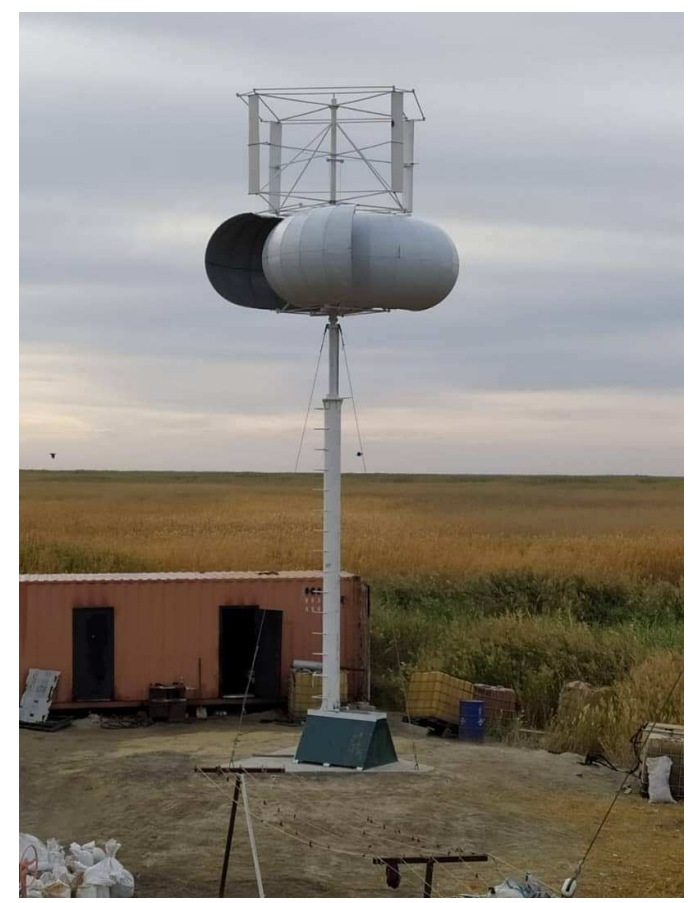

Figure 1: Wind Power Station.

However, as tests have shown, only sail blades operate at low wind speeds, and aerodynamic blades do not significantly contribute to the electric-power generation. Therefore, there remains a need to increase the efficiency factor, utilization ratio over time, and rated power utilization ratio, i.e. it is necessary to enhance the efficiency of operation both at low wind speeds and at higher speeds. At the same time, practically uniform power output should be ensured regardless of wind speeds and directions.

It is possible to solve this problem in the following way (Figure 2) [17]. The vertical axis wind power station contains sail blades in the form of a hollow torus and wide aerodynamic blades, which significantly increases the efficiency of the wind power station at low wind speeds because at low wind speeds aerodynamic blades operate like sail ones. The ability to change the blade-swept area by closing and opening the sail and aerodynamic blades extends the range of wind speeds used, i.e. the utilization ratio of wind energy over time. 


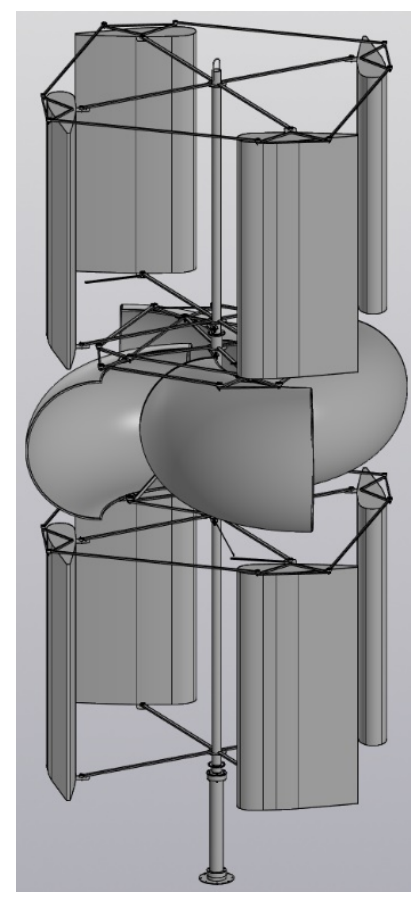

Figure 2: Sail Aerodynamic Wind Power Station.

For example, as wind speed increases, the sail blades close due to wind pressure, the aerodynamic blades reset to a normal position and begin to operate only as aerodynamic ones. This increases the efficiency factor. As the wind becomes stronger, the sail blades close, and the aerodynamic blades deflectfrom their axis and resist rotation. This prevents the acceleration of rotation, which ensures the reliability of the wind power station and increases the utilization of wind energy over time, i.e. the wind power station will operate efficiently at speeds ranging from 3 to $50 \mathrm{~m} / \mathrm{sec}$. As a result, wind power station is able to operate during longer days, thus augmenting electric power output. But what is more important is that the enhanced parameters of the blades help achieve rated power at low wind speeds.

It is also possible to significantly raise the unit capacity of the station without compromising its reliability. This can be achieved due to the fact that at low wind speeds, for example, at speeds up to $8-10 \mathrm{~m} / \mathrm{sec}$, the blade-swept area increases due to the opened sail blades and the use of wide aerodynamic blades with a concave inner side that in the meantime operate like sail ones. As the wind speed rises, for example, up to $20-25 \mathrm{~m} / \mathrm{sec}$, the blade-swept area of the sail blades decreases due to their closure, and the aerodynamic blades reset to a normal position. At gusts of wind over, let's say, $20-25 \mathrm{~m} / \mathrm{sec}$, the wind pressure closes the sail blades completely.

The operational reliability is ensured by the design simplicity and due to the fact that the blade-swept area increases as the wind speed decreases and decreases as the wind speed increases. And with a significant increase in wind speed, aerodynamic blades resist rotation preventing an increase in the number of revolutions.

\section{RESULTS AND DISCUSSIONS}

The practice has shown that when the blades are closed, the wind power station continues to function for the following reason. When blades are closed, the wind resistance decreases, the blades begin to open, but because of the strong wind the blades close again and continue to rotate.

To determine the number of blades and the possibility of using the concavity of the inner side of the aerodynamic 
blades, experiments were conducted on a physical model (Figure 3) with three, four, and five blades. The results are summarized in Tables 1 and 2.

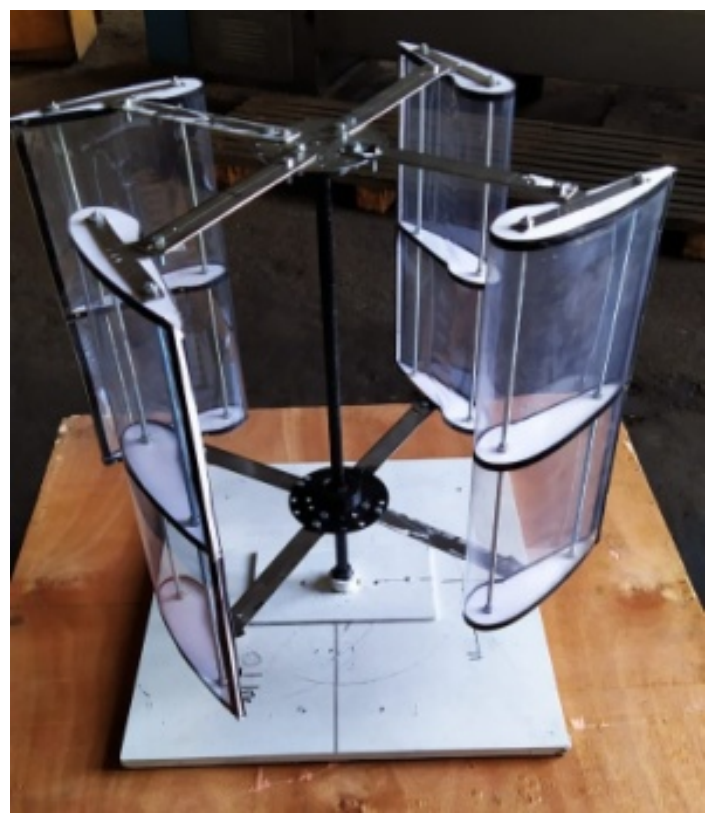

Figure 3: WPS Model.

It should be noted that the traditional drop-shaped blades on both sides started rotating at a higher wind speed than the blade with the concave inner side. Thus, aerodynamic blades with a concave inner side operate like sail ones at low speeds more efficiently than blades with a traditional blade section.

Table 1: Wind Speeds at which the Aerodynamic Blades Start up at various Angles of their Installation inward Relative to the Circumference Circumscribed by the Blades

\begin{tabular}{|l|l|l|l|l|l|}
\hline \multicolumn{1}{|c|}{$0^{0}$} & \multicolumn{1}{c|}{$7^{0}$} & \multicolumn{1}{c|}{$15^{0}$} & \multicolumn{1}{c|}{$25^{0}$} & \multicolumn{1}{c|}{$35^{0}$} & \multicolumn{1}{c|}{$45^{0}$} \\
\hline $\begin{array}{l}\text { Start at } \\
1.1-1.3 \mathrm{~m} / \mathrm{sec}\end{array}$ & $\begin{array}{l}\text { Start at } \\
1.3-1.5 \mathrm{~m} / \mathrm{sec}\end{array}$ & $\begin{array}{l}\text { Start at } \\
1.0 \mathrm{~m} / \mathrm{sec}\end{array}$ & $\begin{array}{l}\text { Start at } \\
0.6 \mathrm{~m} / \mathrm{sec}\end{array}$ & $\begin{array}{l}\text { Start at } \\
1.0-1.3 \mathrm{~m} / \mathrm{sec} \\
\text { and started swinging back and forth }\end{array}$ & $\begin{array}{l}\text { Start at } \\
\text { backward movement }\end{array}$ \\
\hline
\end{tabular}

Table 2: Wind Speeds at which the Aerodynamic Blades start up at Various Angles of their Installation outward relative to the Circumference Circumscribed by the Blades

\begin{tabular}{|l|l|l|l|l|l|}
\hline \multicolumn{1}{|c|}{$0^{0}$} & \multicolumn{1}{|c|}{$7^{0}$} & \multicolumn{1}{c|}{$15^{0}$} & \multicolumn{1}{c|}{$25^{0}$} & \multicolumn{1}{c|}{$35^{0}$} & \multicolumn{1}{c|}{$45^{0}$} \\
\hline Start & & & Start at & Start at & Start at \\
at & Start at & Start at & $1.6-1.7 \mathrm{~m} / \mathrm{sec}$ & $\mathrm{m} / \mathrm{sec}$ \\
$1.1-$ & $1.3-$ & $1.4-1.6$ & $\begin{array}{l}\text { and started swinging back } \\
\text { and forth }\end{array}$ & $\begin{array}{l}\text { and started swinging back } \\
\text { and forth }\end{array}$ & $\begin{array}{l}\text { Start at } \\
1.9-2.0 \mathrm{~m} / \mathrm{sec} \\
\text { backwardmovement }\end{array}$ \\
\hline
\end{tabular}

Tables 1 and 2 made it evident that if there is no wind, the WPS' blades should be installed inwards at an angle of about $25^{\circ}$. This will ensure the start of rotation at the lowest wind speed. The position of the blades will further change depending on the wind speed as described above.

To determine the rational number and position of blades, experiments were performed with the above-mentioned physical models and the results are summarized in Table 3. All the models were identical, the only difference was the number of blades. The resistance to rotation was equal everywhere. 
Table 3 shows that the greatest number of revolutions is achieved with three blades installed outwards at an angle of $0^{0}$ and $7^{0}$ relative to the tangent. At the same time, the rotation starts at an angle of $25^{0}$ inwards, while the number of revolutions at the same angle was significantly lower than with the blades installed outwards at $0^{0}$ and $7^{0}$ (Table 3 )

Table 3: The Number of Revolutions of a wind-Receiving Section Per Minute

\begin{tabular}{|c|c|c|c|c|c|c|c|c|c|c|c|c|}
\hline \multirow{3}{*}{$\begin{array}{c}\text { Number } \\
\text { of } \\
\text { Blades }\end{array}$} & \multicolumn{11}{|c|}{ The head of the blade is installed relative to the tangent at the angle of } & \multirow{3}{*}{$\begin{array}{c}\text { Wind } \\
\text { speed } \\
(\mathrm{m} / \mathrm{sec})\end{array}$} \\
\hline & \multirow{2}{*}{$0^{0}$} & \multicolumn{2}{|c|}{$7^{0}$} & \multicolumn{2}{|c|}{$15^{0}$} & \multicolumn{2}{|c|}{$25^{0}$} & \multicolumn{2}{|c|}{$35^{0}$} & \multicolumn{2}{|c|}{$\mathbf{4 5}^{\mathbf{0}}$} & \\
\hline & & outward & inward & outward & inward & outward & inward & outward & Inward & outward & inward & \\
\hline \multirow{10}{*}{3} & 216 & 230 & & $158^{*}$ & 164 & 75 & 49 & & & & & \\
\hline & 218 & 211 & 198 & 151 & 164 & $0 J$ & 48 & 0 & 28 & 0 & 16 & \\
\hline & 219 & 219 & 110 & & 164 & (1) & 49 & & & & & $4.0-5.2$ \\
\hline & & 173 & 162 & & & & & & & & & \\
\hline & 174 & 191 & 147 & $133^{*}$ & 128 & $62^{*}$ & & & & & & \\
\hline & 179 & (7) & . & $133^{*}$ & 127 & $64 *$ & 12 & 0 & 12 & $-12 * *$ & 0 & \\
\hline & 175 & 155 & 172 & $139 *$ & 110 & $75^{*}$ & & & & & & \\
\hline & 130 & 124 & 117 & $81^{*}$ & 79* & & & & & & & \\
\hline & 128 & 128 & 117 & $79 *$ & $88^{*}$ & 19 & 8 & 0 & 0 & 0 & 0 & 2020 \\
\hline & 128 & 126 & 113 & $83^{*}$ & $95^{*}$ & & & & & & & $2.0=5.2$ \\
\hline \multirow{9}{*}{4} & 182 & 185 & 172 & 125 & 148 & \multirow{3}{*}{$\begin{array}{l}59 \\
49\end{array}$} & 53 & \multirow{3}{*}{0} & 26 & \multirow{3}{*}{$-16^{* *}$} & \multirow{3}{*}{8} & \multirow{3}{*}{$4.8-5.2$} \\
\hline & 183 & 174 & 170 & 124 & 148 & & 52 & & 30 & & & \\
\hline & 190 & 164 & 167 & 147 & 147 & & 53 & & 29 & & & \\
\hline & 156 & 158 & 149 & 109 & 120 & \multirow{3}{*}{53} & 26 & \multirow{3}{*}{0} & \multirow{3}{*}{15} & \multirow{3}{*}{$-22 * *$} & \multirow{3}{*}{0} & \multirow{3}{*}{$3.8-4.2$} \\
\hline & 150 & 136 & 128 & 93 & 121 & & 23 & & & & & \\
\hline & 150 & 136 & 144 & 106 & 121 & & 18 & & & & & \\
\hline & 114 & 99 & 98 & & 98 & & & & 24 & & & \\
\hline & 115 & 110 & 110 & 144 & 95 & 52 & 17 & 0 & 19 & $-8 * *$ & 7 & \\
\hline & 116 & 94 & 96 & 144 & 97 & 30 & & & 16 & & & $2.8-3.2$ \\
\hline \multirow{9}{*}{5} & 161 & 154 & 159 & 107 & 148 & 51 & 52 & \multirow{3}{*}{0} & 40 & \multirow{3}{*}{$\begin{array}{l}-49 * * \\
-52 * *\end{array}$} & 27 & \multirow{3}{*}{$4.8-5.2$} \\
\hline & 163 & 159 & 160 & 106 & 141 & 46 & 51 & & 36 & & 24 & \\
\hline & 162 & 156 & 160 & 104 & 142 & 41 & 50 & & 37 & & 24 & \\
\hline & 133 & 130 & 132 & 89 & 120 & 41 & 33 & \multirow{3}{*}{$-23 * *$} & 26 & & 18 & \\
\hline & 135 & 132 & 129 & 93 & 115 & 48 & 33 & & 27 & $-59 * *$ & 15 & \\
\hline & 133 & 126 & 132 & 93 & 124 & 47 & 35 & & 28 & & 15 & $3.0^{-4} .2$ \\
\hline & 108 & 105 & 105 & 67 & 90 & & 27 & & 22 & & 20 & \\
\hline & 105 & 107 & 110 & 67 & 85 & 0 & 27 & $-10^{* * *}$ & 24 & 21 & 16 & 2820 \\
\hline & 101 & 105 & 99 & 68 & 90 & & 27 & & 26 & & 19 & $2.0-5.2$ \\
\hline
\end{tabular}

*Starts up with additional impact; **Reverse rotation.

Figure 4 represents graphs of changes in the number of revolutions depending on the wind speed, the number of blades and the minimum speed at which rotation begins depending on the angle of installed blades relative to the tangent circle described by the blades. 


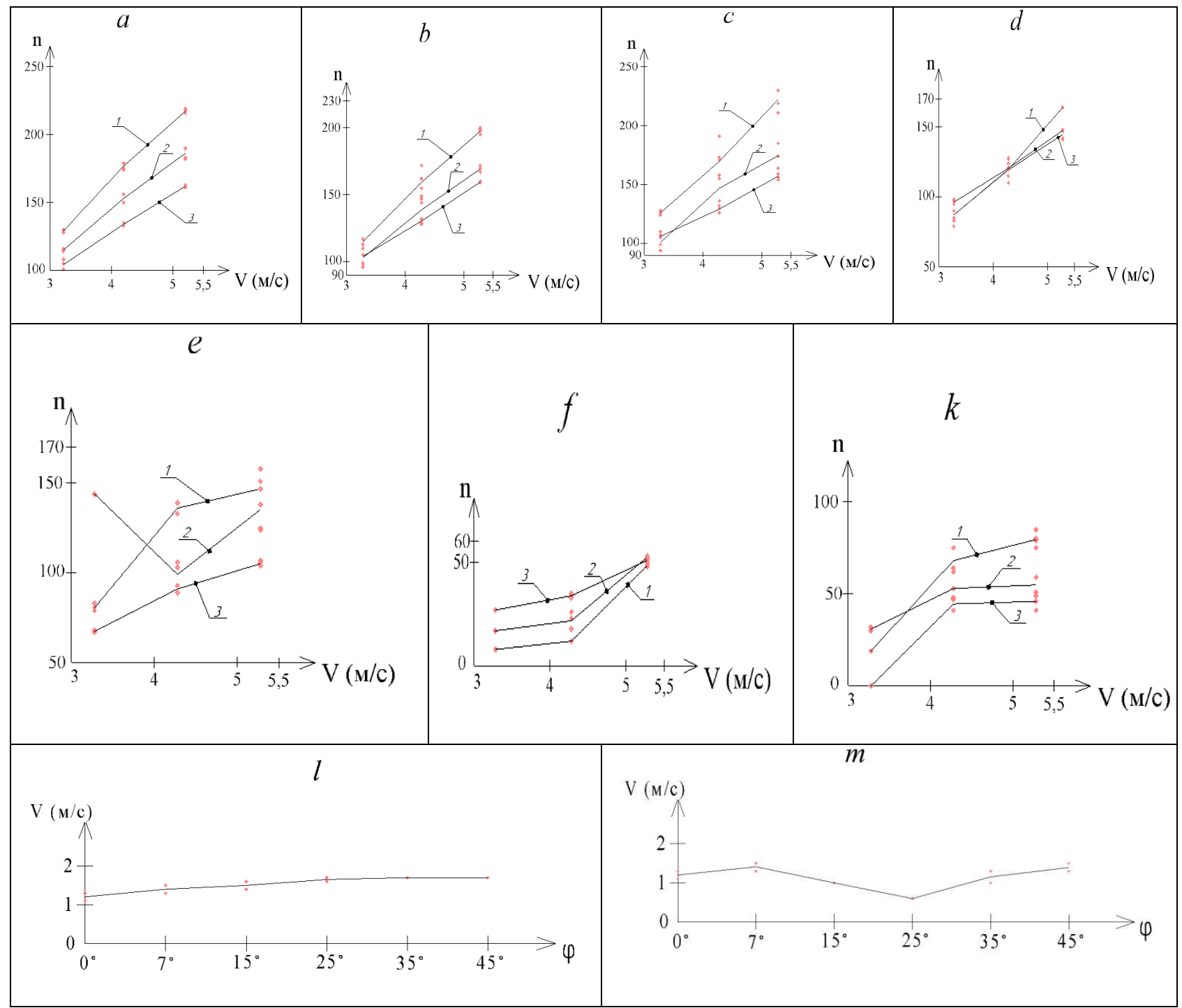

1 - Three-blade wind-receiving section; 2 - Four-blade and 3 - Five-blade wind-receiving section; $\boldsymbol{a}$ - Horizonal axis of blades is perpendicular to the radius; $\boldsymbol{b}$ - Heads of blades are installed inward at $7^{0}$ relative to the tangent circle circumscribed by the blades; $\boldsymbol{c}$ - Heads of blades are installed outwards at $7^{0}$ relative to the tangent; $\boldsymbol{d}$ - Heads of blades are installed inwards at $15^{\circ}$ relative to the tangent; $\boldsymbol{e}$ - Heads of blades are installed outwards at $15^{0}$ relative to the tangent; $\boldsymbol{f}$ Heads of blades are installed inwards at $25^{\circ}$ relative to the tangent; $\boldsymbol{k}$ - Heads of blades are installed outwards at $25^{\circ}$ relative to the tangent; $\boldsymbol{l}$ - Heads of blades are installed outwards; $\boldsymbol{m}$ - Heads of blades are installed inwards; $\boldsymbol{\varphi}$ - Position of blades relative to the tangent.

\section{Figure 4: Graphs of changes in the Number of Blades' Revolutions Depending on their number and the wind speed} $(a, b, c, d, e, f, k)$, and the Minimum Speed at which rotation $(l, m)$ begins

It follows there from that in the absence of wind, the sail blades are fully open, and the aerodynamic blades are partially closed, i.e. their head part is turned inward at $25^{\circ}$. At low speeds, the open sail blades and wide aerodynamic blades are exposed to wind pressure, the wind receiving part starts rotation, which is transmitted through the shaft to the electromechanical part.

As wind speed increases, the sail blades begin to close due to the wind pressure, and the aerodynamic blades start 
to deflect. As the wind continues to get stronger, the sail blades close even more, and the aerodynamic blades reset to a normal position and begin to operate like aerodynamic blades.

With a significant increase in wind speed, the sail blades close and deflect the aerodynamic blades to the position where they are deflected outwards and begin to resist rotation, i.e. to brake it, which prevents the wind-receiving section of the WPS from increasing the speed of rotation. This increases the range of wind speeds used.

As the wind speed weakens, the pressure on the sail blades decreases, as a result of which they open up. This in turn resets the aerodynamic blades to their initial position.

\section{CONCLUSIONS}

In general, at low wind speeds, aerodynamic blades and sail blades operate like sail ones. When the wind intensifies, aerodynamic blades operate like aerodynamic one. When the wind is strong, the sail blades partially close reducing the blade-swept area, and the aerodynamic blades switch into braking mode.

\section{ACKNOWLEDGEMENT}

The authors express appreciation to Zhanabayev E., Nafikov R.T. and Buktukov R.B. for the production of physical models and assistance in experimental works.

\section{REFERENCES}

1. Carsten A.D. (2017).The Kazakhstan Future Energy Transition.How Germany May Contribute. Proceedings of the World Congress of Engineers and Scientists WSEC-2017.Astana. Vol. 1, 43-49.

2. “Rotor with Vertical Shaft and Revolution Adjustment of the Wind Power Station" (FRG Patent DE 102004031105 Al).

3. Reddy, P. RAVINDER, and M. Saikiran. "Aerodynamic Analysis of Return Channel Vanes in Centrifugal Compressors." International Journal of Mechanical Engineering (IJME), 5 (1), 7382 (2016).

4. “Modified Savonius Rotor" (USA PatentUS 6,283,711 B1).

5. "Vertical Axis Turbine" (UK Patent GB 2420597 A).

6. Buktukov N.S., MoldabayevaG.Zh., BuktukovB.Zh.\&Zhakyp A.K. Wind Power Station. RK Patent No. 31790.

7. Pittala, Suresh, and T. Diriba. "Computational Fluid Dynamics Analysis of Impeller Design For A Pump." International Journal of Mechanical Engineering (IJME) 5.4.

8. Lordkipanidze M.M. \&Jamarjashvili V.A. (2017). Innovation technologies and projects of green energetics.Proceedings of the World Congress of Engineers and Scientists WSEC - 2017.Astana. Vol. 2, 62-67.

9. Web page sam-posebe.blogspot.com/2012/10/blog-post.html?m=1

10. National Program for Development of Wind Industry in the Republic of Kazakhstan until 2015 with an Outlook Until 2024.

11. VISWAM, RAHUL, and S. SANKAR. "PROFILE MODIFICATION OF WIND TURBINE BLADE FOR IMPROVING EFFICIENCY." IMPACT: International Journal of Research in Engineering \& Technology (IMPACT: IJRET). 3. 12, Dec 2015, 49-58

12. Vision and Strategy for European Electricity Networks of the Future. -European Commission, 2016.

13. Impact of Wind Power Generation in Ireland on the Operation of Conventional Plant and the Economic Implications. - ESB 
National Grid, 2014.

14. EPIA-Global-Market-Outlook-for-Photovoltaics-until-2015.

15. Bezrukih P.P., ArbuzovYu.D., Borisov G.A. \&VissarionovV.I. (2012). Resources and Efficiency of Renewable Energy Sources in Russia. Saint Petersburg: Nauka.

16. CHERFIA, NAIM, DJALLEL KERDOUN, and ABDERRAOUF BOUMASSATA. "Correction of the mechanical speed for the dfig wind turbine." International Journal of Research in Engineering and Technology 2.11 (2014): 29-38.

17. Dakalov M.V. (2013). Statutory Regulation of Renewable Energy Sources Use in the USA: Basic Documents. Business in Law, 1, 224-226.

18. Dakalov M.V. (2013). Statutory Regulation of Renewable Energy Sources Use in the EU.Science and Business: Ways of Development, 1(19), 58-60.

19. Jang Y.-S., Lee D.-J. \& Oh H.-S.(2013). Evaluation of New and Renewable Energy Technologies in Korea Using Real Options. Int. J. Energy Res.. 37(13), 1645-1656.https://doi.org/10.1002/er.2985

20. Bayeshov A.B., Yegeubayeva S.S. \&Bayeshova A.K. (2016). Current Status of the Transition to Alternative Energy Sources.Reports of the National Academy of Sciences of the Republic of Kazakhstan, 2(306),82-88.

21. Buktukov N.S., MoldabayevaG.Zh., BuktukovB.Zh. \&Zhakyp A.K.Wind Power Station. RK Patent No. 31791. 

